\title{
INFLUENCE DE TROIS FACTEURS SUR LE PEUPLEMENT D'OECOPHYLLES DANS LA LUTTE BIOLOGIQUE CONTRE LA PUNAISE DU COCOTIER EN BASSE CÔTE D'IVOIRE
}

\author{
K. ALLOU1', M. DOUMBIA² etH. ATTADIALLO² \\ ${ }^{1}$ CNRA, Station de Recherche Marc Delorme 07 BP 13 Abidjan 07 \\ 2UFR-SN. Université d'Abobo-Adjamé 02 BP 801 Abidjan 02
}

\begin{abstract}
RESUME
Pseudotheraptus devastans DISTANT cause d'importants dégâts localisés sur les noix de cocotiers en Côte d'Ivoire. Une lutte biologique a été conduite contre cet insecte avec l'utilisation d'un ennemi naturel connu sous le nom Oecophylla longinoda LATREILLE. Le peuplement d'oecophylles sur les cocotiers est accéléré par la construction de ponts entre arbres peuplés et arbres non colonisés. Par ailleurs, les recrûs forestiers ont permis de doubler le peuplement en oecophylles des parcelles non entretenues. Par contre, les parcelles régulièrement entretenues ont vu réduire leur peuplement. Les fourmis du genre Camponotus MAYR ont un effet antagoniste. Elles empêchent l'installation de Oecophylla longinoda par leur présence sur les cocotiers. Pour réussir les recolonisations des arbres déjà occupés par Camponotus, il faut préalablement faire un traitement chimique localisé. La saison sèche favorise l'activité protectrice de Oecophylla longinoda, qui se manifeste par un fort taux de peuplement en oecophylles. En saison pluvieuse par contre, ce taux est faible.
\end{abstract}

Mots clés : Cocotier, lutte biologique, Oecophylla longinoda, Pseudotheraptus devastans, Camponotus sp, Côte d'Ivoire.

\begin{abstract}
EFFECT OF THREE FACTORS ON OECOPHYLLA POPULATION IN THE BIOLOGICAL CONTROL OF THE COCONUT TREE COREID BUG IN SOUTHERN COTTE D'IVOIRE.
\end{abstract}

Pseudotheraptus devastans DISTANT is the cause of important localized damages on coconuts in Côte d'Ivoire. Biological control of this insect was conducted with the use of a natural enemy called Oecophylla longinoda LATREILLE. The population of oecophylla on coconut trees was accelerated with the construction of bridges between colonized and non-colonized trees. Moreover, the forest regrowth allowed the oecophylla population to be doubled in uncleaned plots. However, in regularly cleaned plots, the population was considerably reduced. Black ants of the genus Camponotus MAYR had an antagonistic effect on red ants. These black ants didn't allow the establishment of Oecophylla longinada when present on the coconut trees. For the recolonization of trees to be succesful already colonized, a localized chemical treatment must be applied beforehand. The dry season was found to favour a protecting potential of Oecophylla longinoda. This protection was shown by the high population rate of oecophylla. On the other hand, during the rainy season, this rate was found to be low.

Key words : Coconut tree, biological control, Oecophylla longinoda, Pseudotheraptus devastans, Camponotus sp., Côte d'lvoire.

\section{INTRODUCTION}

Pseudotheraptus devastans DISTANT est un hémiptère piqueur et suceur de la famille des Coreidae. Sur le cocotier (Cocos nucifera L.), les larves et les adultes, piquent les jeunes noix de moins de 9 mois (Mariau et al., 1981) aux abords des pièces florales (pétales et sépales) en leur injectant une salive (sécrétions de glandes labiales) contenant des substances 
toxiques qui provoquent très tôt la lyse des tissus touchés. Une seule noix peut être l'objet de plusieurs attaques. Les points de piqûres forment une ou plusieurs couronnes caractéristiques. Selon le nombre d'attaques reçues, le développement de la jeune noix peut être interrompu (chute de noix immatures) ou contrarié avec une teneur en coprah réduite (Mariau, 1969). Ces dégâts entraînent une perte de production de 50 à $80 \%$ d'où la nécessité de lutter efficacement contre ce ravageur afin de maintenir la population en permanence en dessous du seuil de nuisibilité économique qui est de 30 insectes à l'hectare (Allou, 2000).

La lutte chimique donne de bons résultats mais, elle n'est pas économiquement rentable car les traitements sont répétés tous les mois avec aussi le risque de voir apparaître une résistance chez ces insectes (Julia et Mariau, 1978). La lutte biologique à l'aide de la fourmi rouge, Oecophylla longinoda LATREILLE (Hymenoptera; Formicidae) a montré qu'une parcelle colonisée (70 \% d'oecophylles) a baissé à moins de $10 \%$ les dégâts de la punaise (Douaho, 1984 ; Fataye et de Taffin, 1989).

Néanmoins, l'implantation de ces fourmis se trouve confrontée à trois facteurs : le climat, les recrûs forestiers et les ennemis naturels dont les fourmis noires du genre camponotus. Cette étude menée de 1998 à 2001 se propose de trouver les conditions facilitant la colonisation des parcelles en oecophylles.

\section{MATERIEL ET METHODES}

\section{MATERIEL}

\section{Fourmis rouges ( $O$. longinoda)}

Les fourmis rouges sont des insectes sociaux dont les colonies se caractérisent par un polymorphisme composé de 3 types d'individus (castes).

Les reines : ce sont des femelles fertiles. Ailées au début de leur vie imaginale, elles deviennent aptères après la fécondation (au cours d'un vol nuptial).

Les mâles : ceux-ci sont toujours ailés au cours de leur brève vie imaginale. Ils meurent peu après le vol nuptial ;
Les ouvrières : celles-ci toujours aptères sont les individus les plus nombreux de la colonie. Ce sont des mâles non fertiles. Elles s'occupent de la vie, de l'entretien et de la défense de la colonie. Ce sont des prédateurs très actifs de nombreux autres insectes. Elles sont très agressives à l'égard de tout étranger à la colonie. La survie d'une colonie dépend exclusivement de la présence de la reine. Une seule colonie peut s'étendre sur plusieurs arbres à la fois.

\section{Cocotier}

Les observations ont été faites sur différentes parcelles abritant plusieurs variétés (Hybrides, Nains et Grands) de cocotiers qui sont réceptifs aux oecophylles dès le planting. La présence des fourmis est importante pour protéger les premières productions obtenues dès la $5^{\mathrm{e}}$ année. La colonisation de ces arbres par les fourmis débute à 4 ans, jusqu'à 10 à 15 ans, c'est-àdire lorsque les palmes sont hors de la portée des mains.

\section{METHODES}

\section{Installation des nids d'oecophylles}

Les nids de fourmis rouges sont récoltés avec les reines très tôt le matin sur les vieilles parcelles. Ils sont déposés sur les cocotiers à coloniser en prenant soins de mettre une même colonie sur un seul arbre. 3 à 4 mois après l'installation des colonies, il faut procéder à un premier relevé de contrôle. A partir de ce relevé, le pourcentage en oecophylles est déterminé à partir de la formule suivante :

Peuplement en oecophylles $(\%)=$ Nombre d'arbres peuplés $\times 100$ / Nombre d'arbres vivants.

A partir du taux de peuplement, le taux de variation, qui est la différence entre le taux de peuplement récent et le taux de peuplement précédent, est calculé.

Si pour le premier relevé, le taux de peuplement en oecophylles est inférieur à $50 \%$, il faut procéder à un $2^{e}$, puis éventuellement à un $3^{e}$ transfert, suivis à chaque fois de relevés de contrôle 3 ou 4 mois plus tard. Lorsque le seuil de $50 \%$ est atteint, de nouvelles colonies à partir, des anciennes, peuvent naturellement se 
créer et porter le taux de peuplement à 70 voire $80 \%$. Pour favoriser cette extension horizontale des colonies, les feuilles de différents arbres qui ont été séparées, sont jointes afin de constituer des ponts artificiels.

\section{Influence sur le peuplement des oecophylles}

\section{Entretiens des parcelles}

Deux essais ont été mis en place : le premier en janvier 2000 sur sept parcelles $(023,050$, 051, 091, 092, 084 et 115). Après l'installation des oecophylles, deux parcelles (092 et 091) ont été entretenues régulièrement à raison d'un passage par mois soit de janvier à mai 2000. Les autres parcelles (023, 050, 051, 084 et 115) sont restées sans entretien sur une période de 5 mois (de mai à septembre 2000).

Le second essai en octobre 2000, a été effectué sur 12 parcelles $(023,043,050,051,064,065$, 084, 091, 092, 074, 094 et 115).

Sept parcelles (051, 065, 084, 091, 092, 094 et 115) sont restées sans entretien, saufs les ronds autour des cocotiers. Les cinq autres parcelles $(023,043,050,074$ et 064$)$ ont été régulièrement entretenues.

\section{Facteurs climatiques}

L'influence de la pluviométrie mensuelle sur le peuplement d'oecophylles a été étudié dans trois essais :

- le premier essai s'est déroulé sur les parcelles 014, 024, 074, 115, 092, 091 et 132 avec des relevés d'oecophylles en mai, juin 1998 et mars 1999 ;

- le second, sur les mêmes parcelles et une autre (032) avec un relevé en novembre 1999;

- le troisième a été réalisé sur les parcelles (023, 043, 050, 051, 084, 091 et 092), et les relevés ont été effectués en mai et juin 2001.

\section{Suivi des fourmis noires}

Dans les parcelles d'observation 023, 043 et 050 , nous avons suivi les cocotiers peuplés en fourmis noires sur plusieurs mois, de 1999 à 2001.

\section{Evolution des colonies d'oecophylles}

Les populations de colonies ont été suivies régulièrement à l'aide de relevés basés sur l'échantillonnage suivant:

- 3 arbres par ligne et une ligne sur 6 ;

- régime 4 très réceptif ou à défaut le régime 3.

Les punaises $P$. devastans sont comptées sur les différents arbres de l'échantillon et le nombre de noix attaquées. Les données obtenues permettent de déterminer l'indice de population (I) selon l'expression ci-après :

$\mathrm{I}($ insectes $/$ ha $)=\mathrm{n} \times \mathrm{D} / \mathrm{N}$, où

$\mathrm{n}=$ nombre total d'insectes,

$\mathrm{D}=$ densité de plantation (arbres/ha),

$\mathrm{N}=$ nombre d'arbres échantillonnés.

Lorsque l'indice de population est supérieur à 30 insectes par hectare, un traitement chimique ou une recolonisation est effectué(e). La recolonisation s'effectue par la construction de ponts entre les arbres contigus à partir d'un arbre ayant une forte colonie d'oecophylles.

\section{RESULTATS}

\section{ENTRETIENS DES PARCELLES}

Les taux de peuplements en oecophylles sur les parcelles 091 et 092 (entretenues régulièrement) présentent un taux de peuplement en oecophylles, respectivement de 79,8 et de $73,6 \%$ pour le premier semestre 2000 contre 76,8 et $76,7 \%$ pour le second semestre de la même année (Tableau 1a). Pour ces 2 parcelles, la variation du taux de peuplement en oecophylles, d'une période à l'autre, est négative, soit $-3 \%$ (Tableau $1 \mathrm{a}$ ).

Pour les parcelles non entretenues, 5 mois après l'installation des oecophylles, la variation est de $5 \%$ sur la parcelle 115 , de $8 \%$ sur la 023 , de $21,8 \%$ sur la 050 , de $27,2 \%$ sur la 051 et de $54,6 \%$ sur la 084 (Tableau 1a). D'une façon générale, le taux de peuplement en oecophylles a nettement augmenté au cours du second semestre. 
Au niveau de l'année 2001, le taux de peuplements en oecophylles, dans les parcelles régulièrement entretenues a diversement varié.

Il a varié de 0,1 à $0,7 \%$ dans les 3 parcelles entretenues et de $-16,5$ à -2,6 \% (Tableau 1b) dans quatre autres.

Dans les parcelles non entretenues, le taux de peuplement a largement augmenté avec une variation de $2,1 \%$ à $18 \%$ (Tableau $1 \mathrm{~b}$ ).
Pour la parcelle 023 (jeunes) non entretenues en 2000 et 2001 , le taux de peuplement à presque triplé $(12 \%$ à $32,4 \%$ en mai 2001) (Tableaux $1 \mathrm{a}$ et $1 \mathrm{~b}$ ). Quant à la parcelle 092 régulièrement entretenue sur les deux années, le taux de peuplement a sensiblement diminué de $76,7 \%$ pour le premier semestre 2000 à $68,1 \%$ pour le mois de mai 2001 (Tableaux $1 \mathrm{a}$ et $1 \mathrm{~b}$ ).

Tableau 1a : Taux de peuplement en oecophylles sur parcelles entretenues et non entretenues de l'année 2000.

Oecophylla population rate in cleaned and uncleaned plots during the year 2000.

\begin{tabular}{|c|c|c|c|c|}
\hline \multirow{2}{*}{ Parcelles } & \multirow{2}{*}{$\begin{array}{l}\text { Etat des } \\
\text { parcelles }\end{array}$} & \multicolumn{2}{|c|}{ Taux de peuplement (\%) } & \multirow{2}{*}{$\begin{array}{c}\text { Taux de } \\
\text { variation (\%) }\end{array}$} \\
\hline & & $1^{\mathrm{er}}$ semestre 2000 & $2^{\mathrm{e}}$ semestre 2000 & \\
\hline 091 & PER & 79,8 & 76,8 & $-3,0$ \\
\hline 092 & PER & 76,7 & 73,6 & $-3,1$ \\
\hline 115 & PNE & 19,3 & 24,3 & 5,0 \\
\hline 023 (jeunes) & PNE & 12,0 & 20,0 & 8,0 \\
\hline 050 & PNE & 16,0 & 37,8 & 21,8 \\
\hline 051 & PNE & 37,6 & 64,8 & 27,2 \\
\hline 084 & PNE & 25,3 & 79,9 & 54,6 \\
\hline \multicolumn{2}{|c|}{ Moyenne } & 38,0 & 53,8 & 15,8 \\
\hline
\end{tabular}

PER : Parcelles entretenues régulièrement

PNE : Parcelles non entretenues

Tableau 1b : Taux de peuplements en oecophylles sur les parcelles entretenues et non entretenues de l'année 2001.

Oecophylla population rate in cleaned and uncleaned plots during the year 2001.

\begin{tabular}{|c|c|c|c|c|}
\hline \multirow{2}{*}{ Parcelles } & \multirow{2}{*}{ Etat des parcelles } & \multicolumn{2}{|c|}{ Taux de peuplement (\%) } & \multirow{2}{*}{$\begin{array}{c}\text { Taux de } \\
\text { variation }(\%)\end{array}$} \\
\hline & & Janv.-mars 2001 & mai 2001 & \\
\hline 051 & PNE & 72,8 & 75,4 & $-2,6$ \\
\hline 065 & PNE & 80,8 & 64,3 & $-16,5$ \\
\hline 084 & PNE & 86,7 & 81,3 & $-5,4$ \\
\hline 091 & PNE & 86,5 & 86,6 & 0,1 \\
\hline 092 & PNE & 72,2 & 68,1 & $-4,1$ \\
\hline 094 & PNE & 52,3 & 52,9 & 0,6 \\
\hline 115 & PNE & 30,8 & 31,5 & 0,7 \\
\hline 023 (jeunes) & PRE & 21,1 & 32,4 & 11,3 \\
\hline 043(jeunes) & PRE & 48,5 & 57,7 & 9,2 \\
\hline 050 & PRE & 49,7 & 67,7 & 18,0 \\
\hline 064 & PRE & 87,7 & 89,8 & 2,1 \\
\hline 074 & PRE & 88,2 & 95,0 & 6,8 \\
\hline \multicolumn{2}{|c|}{ Moyenne } & 57,5 & 66,8 & 9,0 \\
\hline
\end{tabular}

PER : Parcelles régulièrement entretenues

PNE : Parcelles non entretenues

\section{FACTEURS CLIMATIQUES}

\section{Influence du climat}

Les conditions climatiques ayant prévalu de 1998 à août 2001 à la Station Marc DELORME près d'Abidjan, site de l'étude, se caractérisent par un climat équatorial de transition à deux saisons des pluies et deux saisons sèches (Figure 1).
La grande saison des pluies s'étend d'avril à juillet avec une valeur nodale en juin et la petite s'étend d'octobre à novembre.

La grande saison sèche s'étend de décembre à mars, avec une pluviométrie totale variant de 0 à $93,7 \mathrm{~mm}$ selon les années et la petite s'étend d'août à septembre, avec une pluviométrie totale variant de 5,5 à $35,5 \mathrm{~mm}$ (Figure 1). 


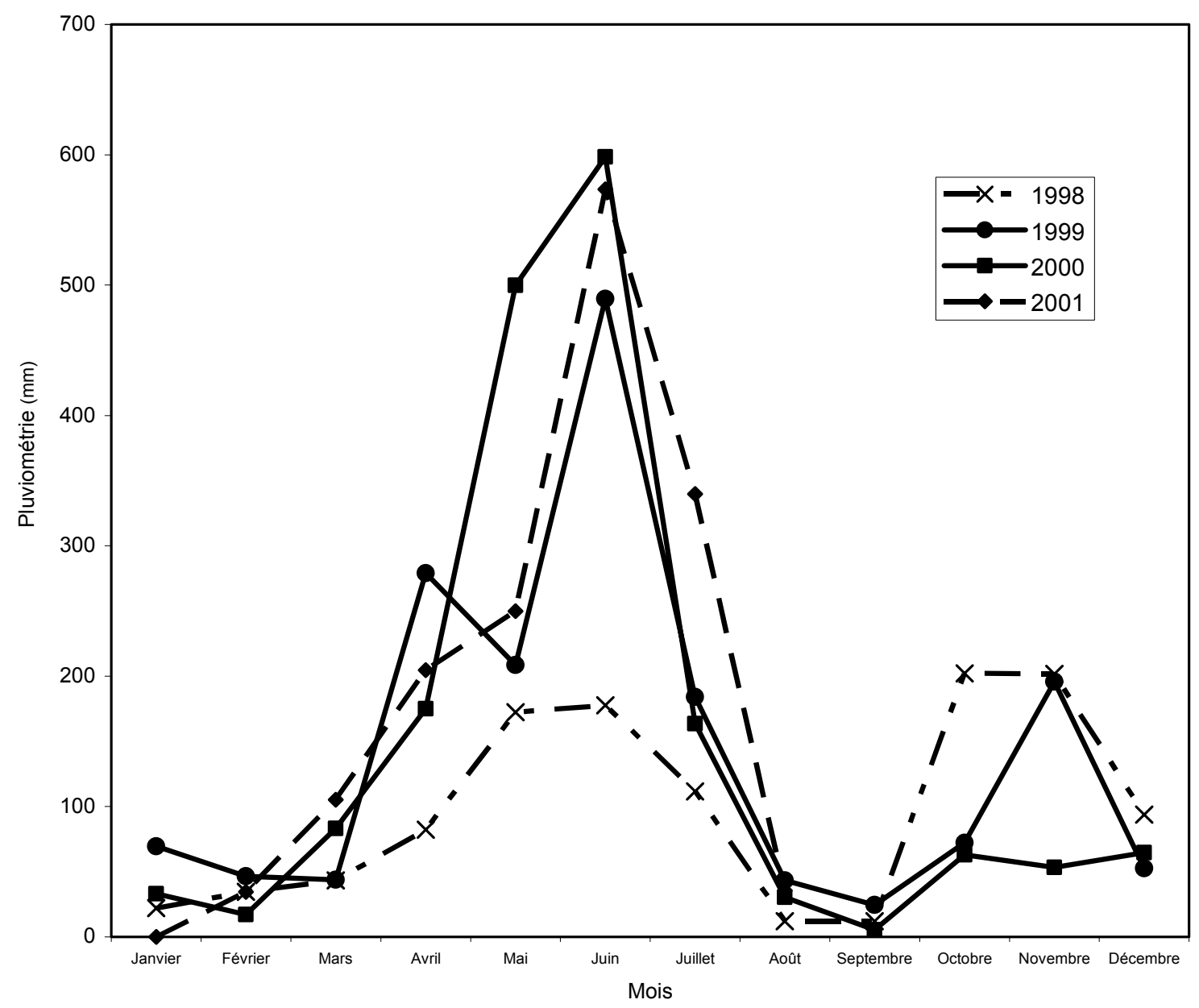

Figure 1 : Pluviométrie mensuelle de janvier 1998 à août 2001.

Monthly rainfall from january 1998 to august 2001.

\section{Comparaison des relevés des saisons pluvieuse et sèche}

Le taux de peuplement d'oecophylles en saison pluvieuse (mai-juin 1998) a varié de 11,6 à $73,3 \%$ (pluviométrie de 172,3 $\mathrm{mm}$ en mai et de $177,8 \mathrm{~mm}$ en juin) (Figure 1). En saison sèche (mars 1999 avec 43,9 mm de pluie), les peuplements d'oecophylles ont été plus importants, avec une augmentation moyenne de 10,3\% (Tableau 2a).

De mars à novembre 1999, on a observé une baisse des peuplements, (de $-25,7$ à $-2,7 \%)$ sur sept des neuf parcelles échantillonnées. Les deux restantes ont eu des variations positives allant de 0,3 à $6,3 \%$ (Tableau 2b). Le taux moyen de mars à novembre 1999 a été de $-4,9$, période au cours de laquelle la pluviométrie a été de $193,9 \mathrm{~mm}$ en novembre contre $43,9 \mathrm{~mm}$ en mars.

\section{Peuplement d'oecophylles en saison pluvieuse}

Les relevés effectués en mai et en juin 2001 (saison des pluies) ont donné respectivement en moyenne $63,6 \%$ et $64,3 \%$ en peuplements d'oecophylles, soit une faible variation de $-0,7 \%$ (Tableau 3 ) et avec une pluviométrie mensuelle de $249,8 \mathrm{~mm}$ en mai et $573,4 \mathrm{~mm}$ en juin (Figure 1 ). 
Tableau 2a : Influence du climat sur le peuplement d'oecophylles en 1998 et 1999.

Climate effect on Oecophylla population in 1998 and 1999.

\begin{tabular}{cccc}
\hline \multirow{2}{*}{ Parcelles } & \multicolumn{2}{c}{ Taux de peuplement (\%) } & \\
\cline { 2 - 3 } & $\begin{array}{c}\text { Mai - Juin 98 } \\
\text { (grande saison } \\
\text { des pluies) }\end{array}$ & $\begin{array}{c}\text { Mars 99 } \\
\text { (grande saison } \\
\text { sèche) }\end{array}$ & $\begin{array}{c}\text { Taux de } \\
\text { variation (\%) }\end{array}$ \\
\hline 014 & 54,1 & 70,1 & 16,0 \\
024 & 68,0 & 83,5 & 15,5 \\
074 & 11,6 & 16,5 & 4,9 \\
115 & 75,3 & 75,2 & $-0,1$ \\
092 & 73,1 & 83,7 & 10,6 \\
091 & 73,3 & 82,5 & 9,2 \\
132 & 54,1 & 70,1 & 16,0 \\
\hline Moyenne & 58,5 & 68,8 & 10,3 \\
\hline
\end{tabular}

Tableau 2b : Influence du climat sur le peuplement d'oecophylles en 1999.

Climate effect on Oecophylla population in 1999.

\begin{tabular}{|c|c|c|c|}
\hline \multirow[b]{2}{*}{ Parcelles } & \multicolumn{2}{|c|}{ Taux de peuplement (\%) } & \multirow{2}{*}{$\begin{array}{c}\text { Taux de } \\
\text { variation } \\
(\%)\end{array}$} \\
\hline & $\begin{array}{c}\text { Mars 99 } \\
\text { (grande saison sèche) }\end{array}$ & $\begin{array}{c}\text { Nov. } 1999 \\
\text { (petite saison de pluies) }\end{array}$ & \\
\hline 014 & 70,1 & 68,0 & $-12,1$ \\
\hline 024 & 83,5 & 57,8 & $-25,7$ \\
\hline 032 & 90,6 & 81,0 & $-9,6$ \\
\hline 074 & 16,5 & 16,8 & 0,3 \\
\hline 091 & 82,5 & 79,8 & $-2,7$ \\
\hline 092 & 83,7 & 74,9 & $-8,8$ \\
\hline 115 & 75,2 & 91,8 & 6,6 \\
\hline 132 & 70,1 & 78,5 & $-8,4$ \\
\hline Moyenne & 71,9 & 76,8 & $-4,9$ \\
\hline
\end{tabular}

Tableau 3 : Influence du climat sur le peuplement d'oecophylles en 2001.

Climate effect on Oecophylla population in 2001.

\begin{tabular}{|c|c|c|c|}
\hline \multirow{2}{*}{ Parcelles } & \multicolumn{2}{|c|}{ Taux de peuplement (\%) } & \multirow{2}{*}{$\begin{array}{c}\text { Taux de } \\
\text { variation (\%) }\end{array}$} \\
\hline & Mai 2001 & Juin 2001 & \\
\hline 023 (jeunes) & 32,4 & 32,7 & 0,3 \\
\hline 0432(jeunes) & 57,7 & 57,4 & 0,3 \\
\hline 050 & 67,7 & 70,3 & 2,6 \\
\hline 051 & 74,0 & 77,9 & 3,9 \\
\hline 084 & 64,3 & 59,5 & $-4,8$ \\
\hline 091 & 81,3 & 82,0 & 0,7 \\
\hline 092 & 68,1 & 70,5 & 2,4 \\
\hline Moyenne & 63,6 & 64,3 & $-0,7$ \\
\hline
\end{tabular}

\section{PONTS D'OECOPHYLLES}

Le taux de succès des ponts réalisés est de 12,2 à $71,4 \%$ après un mois et de 8,3 à $76,1 \%$ après 4 mois (Tableau 4 ). Le meilleur succès est obtenu sur la parcelle 051 pour les deux périodes d'observation. Dans les parcelles 023 et 050 , le taux a régressé respectivement de 33,3 à $8,3 \%$ et de 55,5 à $20 \%$. Par contre, dans la parcelle 115 , il a doublé lorsque la période d'observation est passée de 1 à 4 mois, soit de 12,2 à 26,8 \% (Tableau 4). 
Tableau 4 : Taux de succès des ponts confectionnés par des colonies d'oecophylles.

Rate of success on bridge making by eocophylla population.

\begin{tabular}{cccc}
\hline \multirow{2}{*}{ Parcelles } & \multirow{2}{*}{$\begin{array}{c}\text { Nombre de ponts } \\
\text { confectionnés }\end{array}$} & \multicolumn{2}{c}{$\begin{array}{c}\text { Période d'observation } \\
\text { et taux de succès (\%) }\end{array}$} \\
\cline { 3 - 4 } & & 1 mois & 4 mois \\
\hline 023 & 12 & 33,3 & 8,3 \\
043 & 28 & 64,2 & 60,7 \\
050 & 54 & 55,5 & 20,0 \\
051 & 21 & 71,4 & 76,1 \\
115 & 41 & 12,2 & 26,8 \\
\hline
\end{tabular}

\section{INFLUENCE DES FOURMIS NOIRES}

Les parcelles 043,050 et 023 , où existait une forte colonie de Camponotus sur certains arbres, ont fait l'objet de visites régulières de 1999 à 2001. Sur tous ces arbres, aucune fourmi rouge n'a été observée. De plus, tous les arbres situés à proximité dont les palmes touchaient un arbre peuplé de fourmis noires étaient dépourvus de fourmis rouges. Elles n'ont pu être observées que sur les cocotiers dont les palmes ne touchaient pas les cocotiers peuplés en Camponotus.

\section{DISCUSSION}

Depuis longtemps, les fourmis du genre Oecophylla ont été utilisées comme d'actives prédatrices des punaises ( $P$. devastans) du cocotier.

Différentes études entreprises en Côte-d'Ivoire ont permis de vérifier et de chiffrer l'action de ces fourmis rouges (Mariau et Julia, 1977 ; Julia et Mariau, 1978). Cette colonisation en oecophylles rencontre plusieurs difficultés.

En effet, les recrûs forestiers favorisent l'installation des oecophylles, car ils constituent les relais permettant la colonisation des cocotiers (Stapley, 1971). Par contre, lorsque les parcelles sont bien entretenues, les peuplements d'oecophylles sont moins abondants. II est donc souhaitable, qu'après le transfert de colonies d'oecophylles sur une nouvelle parcelle, d'attendre deux à trois mois avant de commencer l'entretien de celle-ci, afin de donner le temps aux fourmis rouges de s'installer. On remarque que l'évolution du peuplement d'oecophylles est très variable selon les parcelles et selon la période de l'année.
Pendant la saison des pluies, ce peuplement est faible. Pendant cette période, les fourmis sont moins mobiles et se dissimulent dans le feuillage, ce qui rend leur observation difficile. En saison sèche, elles sont en pleine activité et protègent alors les noix contre les attaques de P. devastans (Fataye et de Taffin, 1989).

D'une façon générale en Côte-d'Ivoire, l'occupation de la quasi-totalité des arbres par Oecophylla longinoda est possible entre 4 et 15 ans après la plantation quand les palmes sont encore à la portée des mains. II est alors possible d'effectuer des ponts qui favorisent l'augmentation du taux de peuplements.

La colonisation des cocotiers par des oecophylles peut être réduite par les fourmis noires Camponotus (Fataye et de Taffin, 1989) qui s'opposent à leur installation dans les arbres. Ce fait a été très net dans les parcelles 023 et 050 très colonisées par Camponotus. Leur présence a fait échouer les ponts réalisés entre les arbres en passant d'un taux de peuplement de $33,3 \%$ en 1 mois à $8,3 \%$ en 4 mois sur la parcelle 023 et de 55,5 à $20 \%$ respectivement sur la 050. Ces observations confirment les travaux menés par Julia et Mariau (1978) qui ont montré que les fourmis rouges ne pouvaient pas s'installer sur les arbres fréquentés par les fourmis noires. Pour pouvoir surmonter cette difficulté, il serait utile d'appliquer des traitements insecticides localisés sur le sol ou sur les stipes, de cocotiers afin de favoriser le peuplement des fourmis rouges arboricoles au détriment des camponotus.

\section{CONCLUSION}

Les ouvrières d'oecophylles jouent un rôle actif dans la recherche de la nourriture. Elles sont prédactrices de $P$. devastans pour nourrir les 
larves et les autres membres de la communauté. Une seule et même colonie d'oecophylles peut s'étendre à plusieurs arbres, pourvu que ceuxci se touchent par leurs feuilles, car il faut que tous les nids soient en contact avec la reine. Lorsque le peuplement d'oecophylles est important dans une parcelle donnée, les dégâts causés par les punaises sont faibles. Leur peuplement est favorisé dans les parcelles par les recrûs forestiers où s'organisent de nouvelles colonies.

Il faut éviter de couper les feuilles qui sont en contact avec le sol, car elles constituent des ponts pour les oecophylles passant du recrû à la couronne des cocotiers.

Le développement des oecophylles peut être réduit par des entretiens réguliers après leur implantation, mais aussi, par la présence des fourmis noires du genre Camponotus. Pour réussir les installations d'oecophylles, il faut parfois préparer le milieu en faveur des fourmis rouges par un traitement chimique localisé contre les fourmis noires. Une semaine plus tard, on peut procéder à la recolonisation des arbres.

Les oecophylles sont surtout actifs pendant la période sèche où ils empêchent les punaises de se poser sur les noix. En saison pluvieuse, ils se cachent dans les nids et dans le feuillage devenant ainsi peu actifs.

\section{REMERCIEMENTS}

Les auteurs remercient M. Jean Paul MORIN, chercheur au laboratoire des médiateurs chimiques de l'INRA Versailles (France) pour la lecture et l'analyse critique de cet article.

\section{REFERENCES}

Allou (K.). 2000. Rapport d'activité du premier semestre, opération Défense des Cultures du Programme Cocotier : 1 - 18

Douaho (A.). 1984. Les ravageurs du Palmier et du cocotier, lutte biologique Pseudotheraptus devastans et espèces voisines. Oléagineux, 39 (5) : 557 - 559.

Fataye (A.) et (G.) De Taffin. 1989. Lutte intégrée contre Pseudotheraptus devastans et espèces voisines. Oléagineux, 44 (11) : $325-428$

Julia (J. F.) et (D). Mariau 1978. La punaise du cocotier : Pseudotheraptus sp en Côte d'Ivoire. Etudes préalables à la mise au point d'une méthode de lutte intégrée. Oléagineux, $33(2): 65-72$.

Mariau (D.). 1969 : Pseudotheraptus : Un nouveau ravageur du cocotier en Afrique Occidentale. Oléagineux, 24 (1) : 21-25.

Mariau (D.), (R.) De Desmier, Chenon, (J. F.)Julia et (R.) Phillipe. 1981. Les ravageurs du palmier à huile et du cocotier en Afrique occidentale. Oléagineux, $\mathrm{N}^{\circ}$ spécial, 36(4) : $171-213$.

Mariau(D.), (J. F.) Julia 1977 : Nouvelles recherches sur la cochenille du cocotier Aspidiotus destructor (Sign). Oléagineux, 32 (5) : 217 222.

Stapley (J. H). 1971. La chute prématurée des noix de coco et le rôle complexe des fourmis. Oléagineux, 26 (5) : 317 - 320. 